\title{
"What should happen before asymptomatic men decide whether or not to have a PSA test?" A report on three community juries
}

Chris Degeling PhD, BVSC

\section{Lucie Rychetnik
PhD, $\mathrm{MPH}^{2}$}

Kristen Pickles

Rae Thomas

$\mathrm{PhD}^{3}$

Jennifer A Doust BM BS, PhD, FRACGP

Robert A Gardiner MD, FRCS, FRACS

Paul Glasziou BM BS, PhD, FRACGP

Ainsley J Newson PhD, LLB(Hons), BSc(Hons)

Stacy M Carter $\mathrm{PhD}, \mathrm{MPH}$ (Hons)

1 Centre for Values, Ethics and the Law in Medicine,

University of Sydney, Sydney, NSW.

2 University of Notre Dame Sydney, NSW

$\mathbf{3}$ Centre for Research in Evidence-Based Practice, Bond University, Gold Coast, QLD.

4 The University of Queensland Centre for Clinical Research, Redland City, QLD.

chris.degeling@ sydney.edu.au

doi: 10.5694/mjal5.00164 rostate-specific antigen (PSA) testing of asymptomatic men remains controversial. ${ }^{1}$ Testing may improve prostate cancer survival rates, ${ }^{2}$ but can also lead to harms, such as repeated investigations and the unwanted effects of treatments, including incontinence and impotence. ${ }^{3-5}$ Evidence regarding benefits and harms alone has not resolved tensions over PSA testing. ${ }^{6}$ Disagreement among experts and in guidelines has confused public communication in Australia and internationally. ${ }^{7,8}$

In December 2014, the Prostate Cancer Foundation of Australia (PCFA) and the Cancer Council Australia (CCA) released clinical consensus guidelines for general practitioners for public comment, ${ }^{9}$ after the National Health and Medical Research Council (NHMRC) had published information on the topic for health practitioners. ${ }^{10}$ These documents established criteria for identifying men more likely to benefit than to be harmed by PSA testing. However, it remains unclear if and when GPs should introduce the subject of PSA testing in consultations with individual men. The Royal Australian College of General Practitioners (RACGP) advises GPs not to broach the subject of PSA testing, but to provide full information regarding the benefits, risks and uncertainties of testing and treatment if patients specifically ask about it. ${ }^{11}$

In this article, we report the outcomes of three community juries convened in 2014 to consider the dilemmas associated with PSA testing. A community jury is a group of citizens brought together to receive detailed evidence about a specific problem and to then deliberate on this problem. ${ }^{12}$ Our aim was not to capture the opinions of the broader community, but to ascertain what a well informed citizenry would accept as legitimate PSA testing policy and practice, and

\section{Abstract}

Objectives: To elicit the views of well informed community members on the ethical obligations of general practitioners regarding prostate-specific antigen (PSA) testing, and what should be required before a man undergoes a PSA test.

Design and setting: Three community juries held at the University of Sydney over 6 months in 2014.

Participants: Forty participants from New South Wales, of diverse social and cultural backgrounds and with no experience of prostate cancer, recruited through public advertising: two juries of mixed gender and ages; one all-male jury of PSA screening age.

Results: In contrast to Royal Australian College of General Practitioners guidelines, the three juries concluded that GPs should initiate discussions about PSA testing with asymptomatic men over 50 years of age. The mixed juries voted for GPs offering detailed information about all potential consequent benefits and harms before PSA testing, and favoured a cooling-off period before undertaking the test. The all-male jury recommended a staggered approach to providing information. They recommended that written information be available to those who wanted it, but eight of the 12 jurors thought that doctors should discuss the benefits and harms of biopsy and treatment only after a man had received an elevated PSA test result.

Conclusions: Informed jury participants preferred that GPs actively supported individual men in making decisions about PSA testing, and that they allowed a cooling-off period before testing. However, men of screening age argued that uncertain and detailed information should be communicated only after receiving an elevated PSA test result.

the reasons for their views. Community juries are an established, appropriate method for investigating such questions. ${ }^{12}$ Community juries have been used in Australia and elsewhere to consider questions related to cancer screening. ${ }^{13,14}$ Unlike surveys and focus groups, they involve extensive provision of information, constructive and structured dialogue between ordinary members of the public and experts, and adequate time for consideration of the problem. The process is similar to a legal proceeding, but the outputs are not legally binding; they instead provide evidence for policy making.

We consulted major stakeholders (consumer organisations, GPs, epidemiologists, urologists, the CCA) to design the questions that the juries would consider. All agreed that the key issues to be explored were:
- whether GPs should initiate discussions with asymptomatic men about the PSA test;

- when men should be given information about the potential benefits and harms of testing, biopsy and treatment.

Valid consent for interventions is integral to an ethical health care system, and providing adequate and timely information is fundamental to valid consent. While this has been noted in relation to PSA testing, ${ }^{15,16}$ it is not yet clear what should happen before men decide for or against taking a PSA test. Noting the work currently being undertaken by the NHMRC, CCA and PCFA, we sought information on what selected groups of members of the public consider to be the obligations of GPs regarding informing men about PSA testing, and what else might be required 
before a man could validly consent to a PSA test.

\section{Methods}

Community jury research is a deliberative method, with the following general characteristics:

- a group of citizens is convened for 1 to 3 days;

- they are asked to consider a specific problem;

- they hear evidence from (often opposed) experts, and ask the experts questions;

- they are given time for deliberation and to come to a documented conclusion.

There are two main approaches in community jury research: participants draft open recommendations as a group, or vote on options specified by the researchers. ${ }^{17}$ We used both approaches in our investigation: Jury 1 tested an open approach, while Juries 2 and 3 were asked to vote on specific options (Box 1).

\section{Recruitment and selection}

We recruited three community juries in 2014 - two of mixed gender and ages (Juries 1 and 2), and one of men of PSA screening age (Jury 3) - by placing advertisements and articles in the mass and social media in Sydney. Of 119 respondents, 42 were unavailable on the days scheduled for the juries; 37 with recent personal or close family member experience of prostate cancer treatment, biopsy or active PSA monitoring were also excluded. We sought socioeconomic and cultural diversity for our juries. Juries 1 and 2 were socioculturally diverse but of above-average educational attainment; the all-male Jury 3 was also socioculturally diverse, but its educational attainments broadly matched those of the general Australian population. Forty participants were thus recruited according to their eligibility, sociodemographic characteristics and availability (Box 2).

Each jury commenced with an evening orientation session (Day 0), during which the questions and the jury process were introduced and

1 The questions addressed to the three juries, and the options available for their verdicts

Jury 1 deliberated and drafted recommendations on the open question:

Consent and PSA testing for prostate cancer: "What should happen before men decide whether or not to be tested?"

Juries 2 and 3 were asked to vote on two questions:

Part A. Select 1 or 2:

1. Should GPs introduce the topic of PSA testing during appointments with male patients who have no symptoms?

OR

2. Should they wait until men ask about it?

Part B. Which of these options do you endorse? (Please give your reasons):

1. Men without symptoms should get all the information about the possible benefits and harms of testing, and biopsy and treatment, before they decide whether or not to have a PSA test.

OR

2. Men should not get information about possible benefits and harms of biopsy and treatment before PSA testing. Instead, the doctor should wait until they know the test result. If the test result is raised, then the doctor should give information.

Jurors were asked to endorse either $\mathrm{B} 1$ or $\mathrm{B} 2$, and to give reasons for their decisions. The juries were repeatedly reminded that the questions were specifically about PSA testing for asymptomatic men.

consent was obtained. Jury Day 1 focused on interrogating the evidence and understanding the ethical, legal and practical aspects of the problem. Testimony on the following themes was prerecorded by selected experts and shown to jurors in a video presentation:

- basic biology, diagnosis, treatment and prognosis of prostate cancer;

- qualitative empirical evidence on how Australian GPs manage PSA testing in their practices;

- ethical and legal aspects of patient consent (in general, and with regard to screening);

- potential harms of screening asymptomatic men for prostate cancer; and

- potential benefits of screening asymptomatic men for prostate cancer.

Each presentation lasted about an hour. Prerecording ensured that the evidence presented was standardised, although some experts slightly modified their presentations for Juries 2 and 3 according to the more specific options considered by these juries. The biographical sketches of the experts and the video presentations shown to Juries 2 and 3 are available online. ${ }^{18}$
Immediately after each video, the relevant expert was available for questions through a conference calling system. Facilitated by a researcher, these question-and-answer sessions allowed jurors to clarify or challenge the arguments presented. Facilitation focused on promoting constructive dialogue and fair interaction between jurors. Our observations of unstructured deliberations and the transcripts indicated that this inclusivity was maintained during non-facilitated periods.

For the first hour of Jury Day 2, jurors reflected on, discussed and debated the evidence, aided by a researcher acting as facilitator. Juries then deliberated for an hour without the researchers, and either reached a set of recommendations (Jury 1) or majority verdicts on the questions posed (Juries 2 and 3). The recommendations or verdicts, the underlying reasoning, and dissenting views were reported to the research team in a final, facilitated feedback session.

\section{Data collection and analysis}

The three deliberative groups (juries) were the units of analysis in this study. All jury deliberations (facilitated and non-facilitated) and expert question-and-answer sessions were 
audio-recorded and transcribed. During the final session, the verdicts and reasons were recorded by a researcher on a flipchart. Each point was reviewed by the jury to ensure accuracy. Transcripts were subsequently reviewed to identify key reasons why jurors supported or rejected the presented options.

\section{Ethics approval}

Our study was approved by the Cancer Institute NSW Population and Health Services Research Ethics Committee (HREC/12/CIPHS/46).

\section{Results}

\section{Jury 1}

In response to the question, "What should happen before men decide whether or not to be tested?", Jury 1 recommended that:

- GPs should initiate discussions about PSA testing with 50-70year-old asymptomatic men, and provide information about the limitations of the test and the potential benefits and harms of biopsy and treatment;

- these discussions should be encouraged but not mandatory;

- discussions should inform a man's decision making rather than be constrained by formal procedures (eg, signing a form);

- GPs should consider a cooling-off period, so that men need to wait 1 to 2 days after the discussion before being tested; and

- the community should be informed about expert uncertainty regarding the PSA test, to stimulate discussion between men and their GPs.

Problems discussed by Jury 1 without reaching a consensus were:

- the appropriate content for a patient information sheet;

- how to communicate to men that they can opt out of PSA testing; and

- whether to discourage testing by charging a fee.

\section{Characteristics of the jury participants}

\begin{tabular}{|c|c|c|c|}
\hline & Jury 1 & Jury 2 & Jury 3 \\
\hline Number & 13 & 15 & 12 \\
\hline \multicolumn{4}{|l|}{ Age } \\
\hline$<40$ years & 2 & 5 & 1 \\
\hline 40-70 years & 10 & 8 & 9 \\
\hline$>70$ years & 1 & 2 & 2 \\
\hline Range, years & $28-70$ & 19-75 & $37-74$ \\
\hline Median, years & 52 & 49 & 57 \\
\hline \multicolumn{4}{|l|}{ Gender } \\
\hline Male & 9 & 9 & 12 \\
\hline Female & 4 & 6 & 0 \\
\hline \multicolumn{4}{|l|}{ Highest educational attainment } \\
\hline High school & 2 & 3 & 1 \\
\hline Trade or diploma & 0 & 1 & 7 \\
\hline Bachelor degree & 4 & 7 & 3 \\
\hline Postgraduate degree & 7 & 4 & 1 \\
\hline \multicolumn{4}{|l|}{ Cultural background/ethnicity* } \\
\hline Australian & 11 & 11 & 7 \\
\hline Southern/eastern European & 0 & 1 & 0 \\
\hline Southeast Asian & 1 & 0 & 1 \\
\hline Northeast Asian & 1 & 2 & 2 \\
\hline Southern/central Asian & 0 & 1 & 1 \\
\hline Northwest European & 0 & 0 & 1 \\
\hline \multicolumn{4}{|l|}{ Socioeconomic status of suburb ${ }^{\dagger}$} \\
\hline Low & 1 & 1 & 2 \\
\hline Middle & 1 & 4 & 4 \\
\hline High & 11 & 10 & 6 \\
\hline
\end{tabular}

* Based on the Australian Standard Classification of Cultural and Ethnic Groups (ASCCEG). ${ }^{22}$ †Based on Socio-Economic Indexes for Areas (SEIFA). ${ }^{23}$

\section{Juries 2 and 3, part A}

Similar to Jury 1 , the majority view of both Juries 2 and 3 was that GPs should introduce the topic of PSA testing to asymptomatic men aged 50-70 years (Box 3). Prostate cancer was seen as a legitimate health concern for older men, so that PSA testing was an appropriate topic for general health discussions. Jury 3 (all males) also argued that GPs were best placed to inform men about PSA testing, as GPs were a more reliable point of access to medical advice; relying on other information sources would be "leaving it to chance". All men, they said, should have equal access to the same information.

A minority in both Juries 2 and 3 voted that GPs should not raise the topic of PSA testing with asymptomatic men because other, more important health issues should receive priority, and because men might be more inclined to have a PSA test if GPs raised the topic. They were particularly concerned about the unreliability of the test and the risks of unnecessary treatment ensuing.

\section{Juries 2 and 3, part B}

Like Jury 1, the majority of Jury 2 (mixed gender) voted that detailed benefit-harm information about PSA testing and prostate biopsy and treatment should be provided in advance to support informed decision making. This was a minority position in the all-male Jury 3 (Box 3). 
3 The outcomes of the deliberations of the three juries

\section{Jury 1 recommendations}

GPs should:

- initiate discussions with 50-70-year-old asymptomatic men about PSA testing;

- be prepared to provide men with information about all the potential harms and benefits;

- consider instituting a cooling-off period so that men need to wait before taking the test.

Juries 2 and 3 verdicts

Part A

1. Should GPs introduce the topic of PSA testing during appointments with male patients who have no symptoms?

OR

2. Should they wait until men ask about it?

Jury 2 (mixed gender, $n=15$ ) voted $12-3$ for option 1 ; Jury 3 (all men, $n=12$ ) voted 10-2 for option 1 .

Part B

1. Men without symptoms should get all the information about the possible benefits and harms of testing, and biopsy and treatment, before they decide whether or not to have a PSA test.

OR

2. Men should not get information about possible benefits and harms of biopsy and treatment before PSA testing. Instead, the doctor should wait until they know the test result. If the test result is raised, then the doctor should give information.

Jury 2 (mixed gender, $n=15$ ) voted 13-2 for option 1;

Jury 3 (all men, $n=12$ ) voted $8-4$ for option 2 .

The reasons given by members of Juries 2 and 3 for their views included:

- men have a right to know relevant information before making a decision; and

- after an elevated PSA test result, it might be difficult to refuse subsequent biopsy and treatment, and men may not obtain the information needed to decide about the next steps.

Similar to Jury 1, Juries 2 and 3 supported a cooling-off period so that men could reconsider their decision before testing.

The majority of Jury 2 (13 of 15) supported providing all information before PSA testing. However, 10 of the 13 objected that our wording (especially "should" and "all") was too prescriptive. They wanted GPs to be free to provide information tailored to an individual's level of interest and personal requirements.

Two-thirds of the all-male Jury 3 voted that information about the benefits and harms of biopsy and treatment should be provided only after an elevated PSA test result had been received. These jurors argued that the PSA test alone was not intrinsically harmful, and favoured staggering the delivery of information, with written information available to those who wanted it at any particular point. Jury 3 members, in particular, were concerned about "information overload". They felt that most men would not want to understand the harms and benefits of prostate biopsy and treatment until it was directly relevant to them. They trusted GPs to tell them what they needed to know in a timely manner, avoiding unnecessary anxiety. Notably, some participants argued that details about the risks of biopsies and treatment options should be provided to men by urologists because of their specialist expertise.

\section{Discussion}

After two days of deliberation, all three community juries recommended that GPs should discuss the PSA test with asymptomatic men over 50 years of age as part of routine care. Jurors felt GPs were best placed to consistently inform men about PSA testing, rather than relying on their being informed (or not) by other sources. All three juries wanted GPs, if prompted, to provide information about the limitations, benefits and risks of testing, biopsy and treatment, and to offer to provide more details if desired by the patient. The concept of a cooling-off period to allow men to think about whether or not they wanted a PSA test was also highly valued.

All Jury 3 members were men, and many were having, and appeared committed to, routine annual PSA tests. They also reached different conclusions to the other juries about when information should be provided. While Juries 1 and 2 focused on what would be good for men generally, members of Jury 3 often focused on their own personal experiences and preferences, including a shared inclination to rely on a doctor's assessment of the particular information that was required to inform a patient's decisions. This suggests that, although an informed public prefers GPs to take an active role in educating men about the PSA test, some men of screening age may not wish to be burdened with uncertain and detailed information about the consequences unless they have received an elevated PSA test result.

There are valid reasons why GPs might resist raising awareness of the PSA test. Simply mentioning it may encourage men to favour being tested; patients differ in their information needs; ${ }^{19}$ and communicating the potential harms of PSA screening is difficult. ${ }^{20,21}$ The new consensus recommendations and NHMRCdeveloped information resources promise to support GPs in the challenging task of discussing the topic. Models for communicating information about screening in a balanced and patient-centred way have also been described in the literature. The "consider an offer" model, ${ }^{19}$ for example, suggests that GPs help men consider and evaluate recommendations or offers of screening, while explicitly acknowledging that the 
offer might reasonably be refused. Rather than encouraging screening or expecting people to analyse detailed evidence, whether they felt ready to do so or not, such patientcentred approaches could help individuals decide how much information they wish to receive, and to reflect on their values and preferences regarding benefits and harms when deciding whether or not to be screened.

A limitation to this study is that community juries are comprised of small groups of engaged citizens whose views may not represent those of the general public. However, as all three juries came to similar conclusions, it is likely that our findings are replicable. Our unit of analysis was the deliberative group, but we note that the findings from the all-male jury differed from those of the mixed-gender juries, and that the men in the mixed juries endorsed the final recommendations of the juries in which they participated. This suggests that gender-related factors may influence jury processes.

The juries were clear: GPs should raise the topic of PSA testing and explain the benefits and harms, but tailor their information to the individual patient. Timing of information provision was less clear. PSA testing, the juries concluded, is a health issue that matters to men, and GPs are a reliable, trustworthy source of advice on health issues. These jury outcomes invite critical reflection by professional bodies about how GPs should actively support individual men making decisions about PSA testing.

Acknowledgements: Chris Degeling, Kristin Pickles, Lucie Rychetnik and Stacy Carter received funding from NHMRC project grant \#1023197. Stacy Carter is also supported by an NHMRC Career Development Fellowship (\#1032963) and Paul Glasziou by an NHMRC Australia Fellowship (\#0527500). Rae Thomas, Jennifer Doust and Paul Glasziou received funding from Bond University and NHMRC program grant \#633033.

Competing interests: No relevant disclosures.

c) 2015 AMPCo Pty Ltd. Produced with Elsevier B.V. All rights reserved. 
1 Barry MJ. Screening for prostate cancer - the controversy that refuses to die. N Engl J Med 2009; 360: 1351-1354.

2 Schröder FH, Hugosson J, Roobol MJ, et al. Prostate-cancer mortality at 11 years of follow-up. N Engl J Med 2012; 366: 981-990.

3 Chou R, Croswell JM, Dana T, et al. Screening for prostate cancer: a review of the evidence for the US Preventive Services Task Force. Ann Intern Med 2011; 155: 762-771.

4 Ilic D, O'Connor D, Green S, Wilt TJ. Screening for prostate cancer: an updated Cochrane systematic review. BJU Int 2011; 107: 882-891.

5 Welch HG, Black WC. Overdiagnosis in cancer. J Natl Cancer Inst 2010; 102: 605-613.

6 Brett AS, Ablin RJ. Prostate-cancer screening - what the US Preventive Services Task Force left out. $N$ Engl J Med 2011; 365: 1949-1951.

7 Moyer VA. Screening for prostate cancer: US Preventive Services Task Force recommendation statement. Ann Intern Med 2012; 157: 120-134.

8 Urological Society of Australia and New Zealand. PSA testing policy 2009. http://www.usanz.org.au/uploads/2 9168/ufiles/USANZ_2009_PSA_ Testing_Policy_Finall.pdf (accessed Jul 2015).

9 Prostate Cancer Foundation of Australia and Cancer Council Australia; PSA Testing Guidelines Expert Advisory Panel. Draft clinical practice guidelines PSA testing and early management of test-detected prostate cancer. Sydney: Cancer Council Australia, 2014. http:// wiki.cancer.org.au/australia/Guidelines: PSA_Testing/Guideline_development_ process (accessed Jul 2015).

10 National Health and Medical Research Council. PSA testing for prostate cancer in asymptomatic men: information for health practitioners. 2014. http://www. nhmrc.gov.au/_files_nhmrc/ publications/attachments/men4d_psa testing_asymptomatic_men_140304. pdf (accessed Jul 2015).

11 Royal Australian College of General Practitioners. Guidelines for preventive activities in general practice. 8th ed. Melbourne: RACGP, 2012. http://www. racgp.org.au/your-practice/guidelines/ redbook/ (accessed Jul 2015).

12 Degeling C, Carter SM, Lychetnik L. Which public and why deliberate? - A scoping review of public deliberation in public health and health policy research. Soc Sci Med 2015; 131: 114-121.

13 Rychetnik L, Doust J, Thomas R, et al. Community Jury on PSA screening: what do well-informed men want the government to do about prostate cancer screening - a qualitative analysis. BMJ Open 2014; 4: e004682.

14 Paul C, Nicholls R, Priest P, McGee R. Making policy decisions about population screening for breast cancer: the role of citizens' deliberation. Health Policy 2008; 85: 314-320.

15 Wollin D, Loeb S. Patient perceptions and shared decisions about PSA screening. Rev Urol 2013; 15: 206-207.
16 Knight SJ. Decision making and prostate cancer screening. Urol Clin North Am 2014; 41: 257-266.

17 Lenaghan J, New B, Mitchell E. Setting priorities: is there a role for citizens' juries? BMJ 1996; 312: 1591.

18 Center for Values, Ethics and the Law in Medicine, University of Sydney. Project 5: Prostate screening from the perspective of citizens [website]. 2014. http://cancerscreeningethics.org/ prostate-screening-projects/project-5community-juries (accessed Jul 2015).

19 Entwistle VA, Carter SM, Trevena L, et al. Communicating about screening. BMJ 2008; 337: al591-1593.

20 Howard K, Brenner AT, Lewis C, et al. A comparison of US and Australian men's values and preferences for PSA screening. BMC Health Serv Res 2013; 13: 388.

21 Gattellari M, Ward JE. Will men attribute fault to their GP for adverse effects arising from controversial screening tests? An Australian study using scenarios about PSA screening. J Med Screen 2004; 11: 165-169.

22 Australian Bureau of Statistics. Australian Standard Classification of Cultural and Ethnic Groups (ASCCEG), 2011. http://www.abs.gov.au/ AUSSTATS/abs@.nsf/Lookup/1249. OMain+Features12011?OpenDocument (accessed Jul 2015).

23 Australian Bureau of Statistics. SEIFA 2011. http://www.abs.gov.au/ausstats/ abs@.nsf/mf/2033.0.55.001 (accessed Jul 2015). 